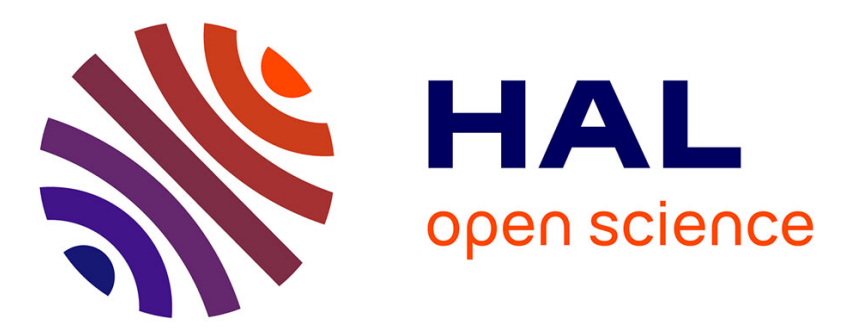

\title{
Mapping the Electromagnetic Near-Field Enhancements of Gold Nanocubes
}

Claire Deeb, Xuan Zhou, R. Miller, S.-K. Gray, Jerome Plain, Sylvie Marguet, G.-P. Wiederrecht, Renaud Bachelot

\section{- To cite this version:}

Claire Deeb, Xuan Zhou, R. Miller, S.-K. Gray, Jerome Plain, et al.. Mapping the Electromagnetic Near-Field Enhancements of Gold Nanocubes. Journal of Physical Chemistry C, 2012, 116 (46), pp.24734-24740. 10.1021/jp304647e . hal-00777236

\section{HAL Id: hal-00777236 https://hal.science/hal-00777236}

Submitted on 13 Dec 2019

HAL is a multi-disciplinary open access archive for the deposit and dissemination of scientific research documents, whether they are published or not. The documents may come from teaching and research institutions in France or abroad, or from public or private research centers.
L'archive ouverte pluridisciplinaire HAL, est destinée au dépôt et à la diffusion de documents scientifiques de niveau recherche, publiés ou non, émanant des établissements d'enseignement et de recherche français ou étrangers, des laboratoires publics ou privés. 


\title{
Mapping the Electromagnetic Near-Field Enhancements of Gold Nanocubes
}

\author{
Claire Deeb, ${ }^{* \dagger, \ddagger}$ Xuan Zhou, $^{\dagger}$ Ryan Miller, $^{\dagger}$ Stephen K. Gray ${ }^{\ddagger}$ Sylvie Marguet, ${ }^{\S}$ Jérôme Plain, $^{\dagger}$ \\ Gary P. Wiederrecht, ${ }^{\ddagger}$ and Renaud Bachelot ${ }^{* \dagger}$ \\ ${ }^{\dagger}$ Laboratoire de Nanotechnologie et d'Instrumentation Optique LNIO-CNRS UMR 6279, Université de Technologie de Troyes, \\ Troyes, France \\ ${ }^{\ddagger}$ Center for Nanoscale Materials, Argonne National Laboratory, Argonne, Illinois 60439, United States \\ ${ }^{\S}$ CNRS-CEA, IRAMIS, SPAM, Laboratoire Francis Perrin, URA2453, 91191 Gif-sur-Yvette, France
}

\begin{abstract}
We imaged and quantitatively characterized electromagnetic hot spots near the surfaces of plasmon resonant gold nanocubes. The strongest fields are localized at the nanocube corners as compared to those on the sides. The near-field enhancement on the surface of the cube was imaged as a function of incident polarization, leading to information on the localization of fields on specific regions on the surface. We found that the field intensity drops dramatically when the nanocube corner is slightly tilted with respect to the incident laser polarization. This dramatic dependence on angle was verified by electrodynamics
\end{abstract} simulations. These results will enable the use of gold nanocubes in field enhancement applications and refractive-index sensing.
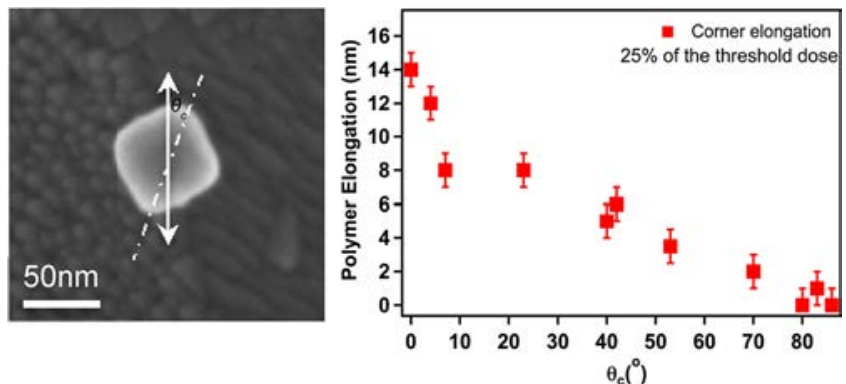

\section{INTRODUCTION}

The optical properties of gold and silver nanocubes (NCs) have been the focus of much recent experimental and theoretical work. $^{1-12}$ Much of this work has focused on the unique properties of the cubes, especially enhanced local electromagnetic fields, particularly at the 8 vertices and along the 12 edges of the cube, when illuminated with light. These hot spots produce a strong optical response, and can enhance interesting phenomena including optical nonlinearities, ${ }^{13}$ fluorescence, exciton-exciton annihilation, ${ }^{14}$ and Raman scattering (i.e., surface enhanced Raman scattering or SERS). ${ }^{2,3,9}$ Although a variety of other nanoparticle geometries can enhance the abovenoted processes, NCs offer advantages in that large, relatively monodisperse colloidal samples can be easily prepared, they are robust, and the size of the NCs can be controlled. Thus, the plasmon resonances of NCs can be tuned across a wide spectrum, while the vertices are expected to give stronger electromagnetic field enhancements than spherical particles.

Various interesting optical effects have been reported on NCs. For example, Sherry et al. used dark-field microscopy to observe a new plasmon resonance effect for a single silver nanocube in which the plasmon line shape has two distinct peaks when the particles are located on a glass substrate. ${ }^{9}$ McLellan et al. utilized silver NCs and correlated the SERS spectra from individual NCs to their physical parameters revealed by high-resolution scanning electron microscopy imaging. ${ }^{2}$ They observed dramatic variations in SERS intensity when the NCs were oriented at different angles relative to the polarization of the excitation laser. ${ }^{2}$ Five years later, the same group also reported a novel approach for the generation of hot spots with sufficiently strong SERS enhancements for singlemolecule detection by simply depositing silver NCs on the surface of a gold or silver substrate. ${ }^{15}$ As a last example, Zhang et al. reported coupling and hybridization of the plasmon modes of a metallic nanostructure by introducing an adjacent semi-infinite dielectric. ${ }^{11}$ They demonstrated that, for a nanocube, a nearby dielectric mediates an interaction between bright dipolar and dark quadrupolar modes, resulting in bonding and antibonding hybridized modes. ${ }^{11}$

To date, little is known about local near-field optical properties of metal NCs, since most of the plasmonic studies were carried out in the far-field. However, to pave the way for tailoring nanostructured electromagnetic fields using NCs, a detailed characterization of their near-field properties is essential. There is considerable theoretical work on the nanostructured field around a nanocube, but there is no direct measurement of the near-field enhancement or its spatial or angular profile. Essentially, these quantities are known only in theory but not in practice. Here, we present a quantitative study of the localized surface plasmons of gold nanocubes in the nearfield. 
In this paper, we map and characterize the electromagnetic field hot spots on individual gold nanocubes (Au NCs) using a novel experimental approach that relies on plasmonic photopolymerization and provides sub-5 nm resolution. ${ }^{16-18} \mathrm{We}$ report field measurements on individual Au NCs with different orientations relative to the excitation laser polarization, leading to information on the localization of fields on specific regions on the surface. We found that the highest fields are localized at the nanocube corners as compared to its sides. The field intensity drops dramatically when the nanocube corner is slightly tilted with respect to the incident laser polarization. This dramatic dependence on angle was verified by electrodynamics simulations.

\section{METHODS}

2.1. Experimental Methods. Nanocube Synthesis and Characterization. Au NCs were synthesized in water following a seed-mediated growth method. NCs of edge length $60 \pm 3$ $\mathrm{nm}$ are prepared following a recently published procedure ${ }^{19}$ based on the earlier experiments of Sau and Murphy ${ }^{20}$ which involves a two-step approach composed of a seeding process and a growth process. The seed solution is prepared by adding an ice-cold $\mathrm{NaBH}_{4}$ solution $(0.42 \mathrm{~mL}, 10 \mathrm{mM})$ into an aqueous solution composed of a mixture of $\mathrm{HAuCl}_{4}(92 \mu \mathrm{L}, 10 \mathrm{mM})$ and CTAB $(7 \mathrm{~mL}, 100 \mathrm{mM})$. This seed solution is kept at 25 ${ }^{\circ} \mathrm{C}$ for $2 \mathrm{~h}$ to decompose excess borohydride before use. The growth solution is prepared by the successive addition of three aqueous solutions: CTAB $(9 \mathrm{~mL}, 22 \mathrm{mM}), \mathrm{HAuCl}_{4}(0.250 \mathrm{~mL}$, $10 \mathrm{mM})$, and ascorbic acid $(3 \mathrm{~mL}, 38 \mathrm{mM})$. Next, $20 \mu \mathrm{L}$ of a water-diluted seed solution (1:50) is injected into the growth solution. The resultant solution is allowed to sit overnight at 25 ${ }^{\circ} \mathrm{C}$. Concentrated solutions of NCs are obtained by centrifugation at $4500 \mathrm{rpm}$ for $15 \mathrm{~min}$, twice, and redispersed in a suitable amount of water. A few $\mu \mathrm{L}$ of these concentrated solutions are dropcast on carbon-coated copper grids and indium tin oxide (ITO) substrates for TEM and SEM measurements, respectively.

Cetyltrimethylammonium bromide $(\mathrm{CTAB} \geq 98 \%)$, chloroauric acid $\left(\mathrm{HAuCl}_{4} \cdot 3 \mathrm{H}_{2} \mathrm{O}\right)$, sodium borohydride $\left(\mathrm{NaBH}_{4}\right.$, 99\%), and ascorbic acid (99+\%) were purchased from Sigma and used as received. Deionized water is used for all experiments and dilutions.

The UV-visible extinction spectra in solution are recorded with a $1 \mathrm{~cm}$ cell on a Jasco V-570 spectrophotometer. Transmission electron microscopy (TEM) observations are made using a Philips CM12 microscope operating at $120 \mathrm{kV}$, and scanning electron microscopy (SEM) images are acquired on a Zeiss-Ultra55-FEG microscope. Figure 1A shows a transmission electron microscope (TEM) image of a concentrated deposit of Au-NCs on a TEM grid. Figure 1B shows a typical scanning electron micrograph (SEM) of $\mathrm{Au}$ NCs well dispersed on the ITO glass substrate. Figure 1C represents the gold cube template that is used for the simulations. Figure 1D shows both the experimental (in red) and theoretical (in blue) extinction spectra of $\mathrm{Au}$ NCs immersed in water. The experimental extinction spectrum is collected on the ensemble solution of $\mathrm{Au}$ NCs immersed in water, while the theoretical spectrum is carried out with an isolated gold cube surrounded by a uniform medium with the index of refraction of water, 1.33. Nonetheless, the level of agreement is quite good, with the overall shapes being very similar and the theoretical maximum $(570 \mathrm{~nm})$ being just slightly to the red of the experimental one $(562 \mathrm{~nm})$.
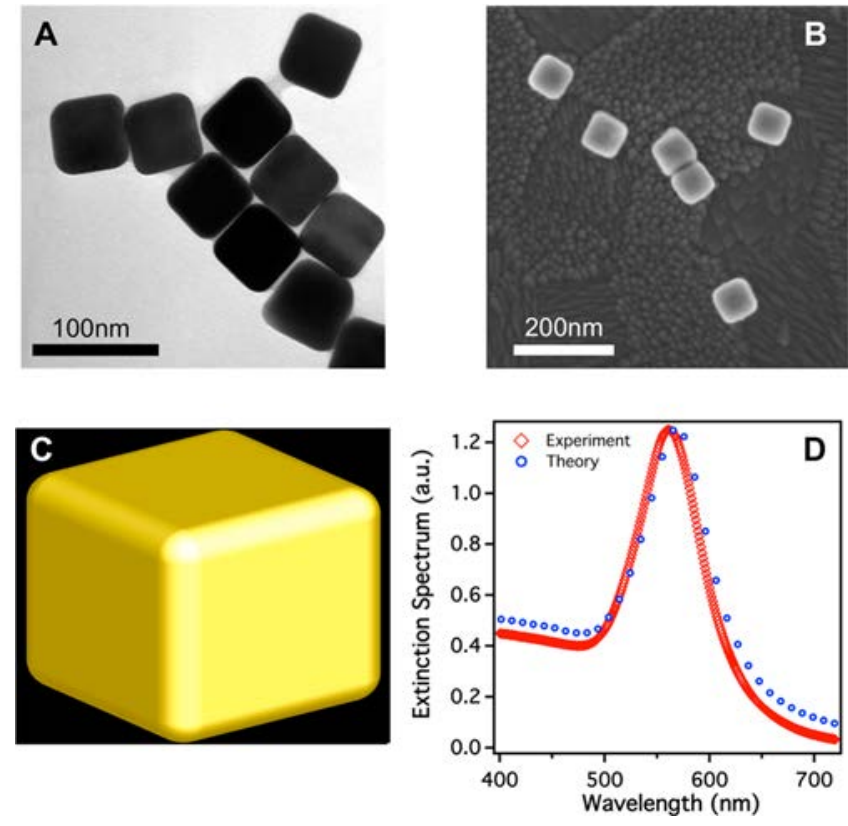

Figure 1. (A, B) Typical TEM and SEM images of Au NCs dispersed on a TEM grid and on an ITO/glass substrate, respectively. (C) A gold cube template used for the simulations. (D) Experimental (in red) and theoretical (in blue) extinction spectra of Au NCs immersed in water.

Near-Field Measurements. The method for measuring the near-field intensities around the nanocube is based on a technique developed in our group in Troyes. It has been shown by Bachelot and co-workers that it is possible to structure a photosensitive polymer solution using the localized surface plasmons of noble metal nanoparticles resulting in a map of the distribution of the near-field intensity around these nanoparticles.

In this technique, we employ a photopolymerizable chemical system that exhibits a threshold dose $D_{\text {th }}$ below which no polymerization can occur. The nonlinear threshold behavior of the formulation allows for high resolution patterning under evanescent excitation of localized plasmons. A full quantitative study as a function of the excitation dose allows us to map the near-field as a function of distance away from the nanostructure. $^{17}$

We contrast the current technique with another method developed in our group. The latter method is based on irradiation of a homogeneous layer of azobenzene based copolymer covering the plasmon resonant nanoparticles with the photopolymer. ${ }^{12,21}$ This approach enables visualization of the optical near-field through local photoinduced displacement of azobenzene molecules experiencing isomerization cycles under laser illumination. Using this chemical system, molecular motions assisted by the localized field have been studied and vectorial imaging of the nanostructured near-field has been achieved. Although this imaging technique constitutes a powerful way to map the different components of the nearfield of noble metal nanoparticles by means of photosensitive molecules, it does not quantify near-field features. The method presented in the current paper is significantly different from that described in refs 12 and 21: It is based on free-radical photopolymerization and allows quantification of the plasmons of metal nanostructures in the near-field, which has been a key challenge in the community. 
Composition of the Photosensitive Solution. The photopolymerizable solution is made up of three basic components: a sensitizer dye, a cosynergist amine, and a multifunctional acrylate monomer, pentaerythritol triacrylate (PETIA). PETIA is used as received from the supplier and forms the backbone of the polymer network. The cosynergist amine is methyldiethanolamine (MDEA), and the Eosin-Y $\left(2^{\prime}, 4^{\prime}, 5^{\prime}, 7^{\prime}\right.$-tetrabromofluorescein disodium salt) is used as the sensitizer dye. This system is developed mainly because of its high sensitivity in the spectral region from 450 to $550 \mathrm{~nm}^{22-24}$ In addition, this liquid system is very flexible, as it is possible to modify the components independently to adjust the physical and chemical properties of the formulation, namely, viscosity, spectral sensitivity, polymerization threshold, and energy. The results reported in this article are obtained with mixtures containing 0.5 wt \% Eosin-Y and 4 wt \% MDEA.

Measurement of the Polymer Elongation. In a typical experiment, we use acetone to clean a glass sheet coated with an optically thin $(3 \mathrm{~nm})$ ITO layer and then make the sheets hydrophilic by means of UV-ozone treatment. A $1 \mu \mathrm{L}$ droplet of a well-diluted $\mathrm{Au} \mathrm{NC}$ solution is then deposited on the surface of the ITO/glass sheet and kept until the solvent evaporates. Once the sample dries, it is washed with a copious amount of acetone, and finally dried with a stream of air. All samples are used within $12 \mathrm{~h}$ after being prepared. After being deposited on the surface, the $\mathrm{Au}$ NCs are precisely characterized using AFM. The photoactive polymer solution is then poured over the sample. We employ normally incident laser light with wavelength $\lambda_{0}=532 \mathrm{~nm}$ from the glass side to photoexcite the system for specific time periods corresponding to a given dose. After application of a dose, the excess polymer solution is poured off. We then use AFM measurements to infer the polymer elongations from the difference of the AFM measurements after and before irradiation. The nature of the measurements is that these elongations are associated with the top ends of the cubes (relative to the bottom glass surface). We focus on the polymer elongation emanating from a corner region and the side region, and in each case consider the elongation along a direction normal to the metal/polymer interface extending away from the cube, and measure these elongations as a function of polarization angle.

Figure 2A shows an AFM image of well-dispersed Au NCs on an ITO surface. The photopolymerizable solution has been designed to meet three requirements: (a) the photoinitiator was chosen to have sensitivity (as evidenced by its absorption spectrum presenting a peak at $532 \mathrm{~nm})^{17}$ in the visible range overlapping the localized surface plasmon resonance (LSPR) spectrum of the nanostructure, (b) the photopolymerizable system should exhibit a nonlinear response based on a threshold dose, below which no photopolymerization can occur, and which needs to be well characterized as a function of the excitation wavelength a priori, and (c) the diffusion of the molecules in the formulation should be ideally low. Indeed, low diffusion and a sharp threshold response of the formulation are crucial for achieving a high resolution of this near-field enhanced photolithography. ${ }^{25}$

During photoexcitation, the laser polarization is fixed linearly, as is indicated by the white arrow in Figure $2 \mathrm{~B}$, and its dose, $D_{0}$, is set at a fraction of the threshold dose, $D_{\text {th }}: f=D_{0} / D_{\text {th }}$. $D_{\text {th }}$ corresponds to the minimum energy necessary to observe formation of polymer on the substrate. ${ }^{16-18}$ Because the exposure dose $D_{0}$ is below $D_{\mathrm{th}}$, the photopolymerization can only be initiated at regions (within a few $\mathrm{nm}$ around the

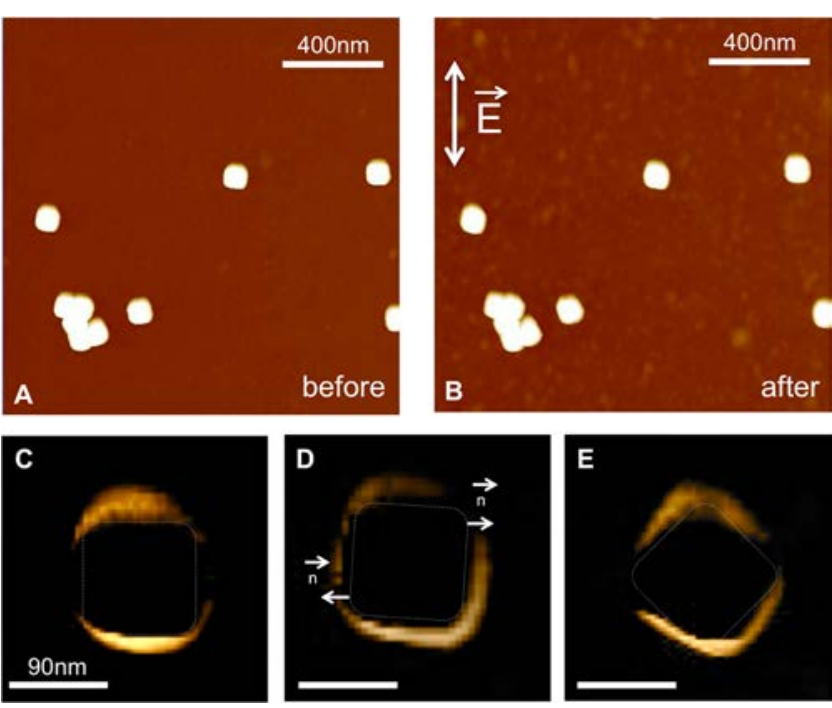

Figure 2. Near-field photopolymerization based on the resonant excitation of $\mathrm{Au} \mathrm{NCs}$ embedded in a photolithographic formulation. (A) Topographic AFM image of well dispersed Au NCs on an ITO surface before photoexcitation. (B) Topographic AFM image of the same region of the sample showing the Au NCs after photopolymerization. (C-E) Differential images of three differently oriented $\mathrm{Au}$ NCs relative to the laser polarization: a cube whose vertical sides are well aligned with the incident polarization (C), a tilted nanocube (D), and a cube with the diagonal axis (corner to corner) parallel to the laser polarization (E). The up-down arrow in panel B represents the incident laser direction. The $90 \mathrm{~nm}$ scale bar shown in panel C applies to all differential images.

nanoparticle) where the near-field enhancement is $1 / f$, or higher. Following a rinsing procedure, an AFM image of the hybrid "nanocube + polymer" system is acquired, as one can see in Figure 2B, from which a background AFM image of the nanoparticle preceding the photoexcitation can be subtracted, leading to differential AFM images such as Figure 2C-E. This differential imaging provides a high signal-to-noise ratio, driftfree and tip convolution-free image of the nanoscale polymer structures fabricated around the nanoparticle. ${ }^{17}$ For instance, in the case of a Au nanocube excited with linearly polarized light, polymer elongation is seen along the polarization direction, while no polymerization is induced where the unit normal vector $\vec{n}$ (represented by the white arrows in Figure $2 \mathrm{D}$ ) is perpendicular to the laser polarization direction. ${ }^{18}$ Parts $\mathrm{C}-\mathrm{E}$ of Figure 2 show three differential images corresponding to three differently oriented $\mathrm{Au}$ NCs relative to the laser polarization. These differential images are highly relevant, since they point out the polymerized zones on the surface of the NCs, and hence the zones where the near-field enhancement contributed to overcome the threshold dose.

The spatial profile of the near-field can be extracted from polymer elongations measured as a function of dose. The local dose $D$ can be expressed by the phenomenological equation ${ }^{17}$

$$
D=F_{\max } D_{0} \exp (-\alpha y)
$$

where $F_{\max }$ is the maximum (at the surface) intensity enhancement factor related to the LSPR, $\alpha$ is the decay constant of the field intensity $\left(\alpha^{-1}\right.$ is its characteristic decay length), and $y$ is the distance from the surface of the metal nanoparticle in the $y$-direction. ${ }^{17}$ 

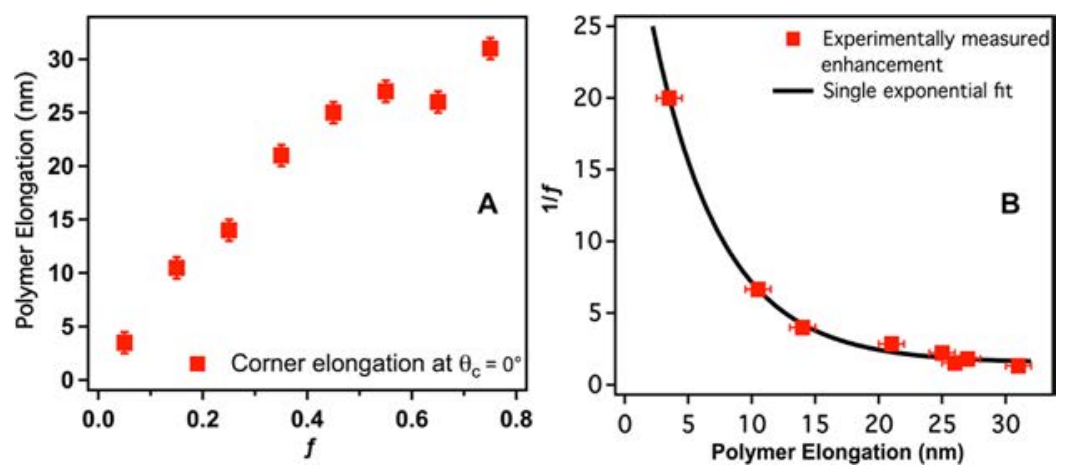

Figure 3. Quantification of the enhancement factor value related to localized surface plasmons. (A) Effect of the exposure dose $f=D_{0} / D_{\text {th }}$ on the photopolymerization elongation of the polymer. (B) Experimental values (red points) of $1 / f$ plotted as a function of the polymer elongation measured by AFM. The black line corresponds to a single exponential fit. Each data point is an average from measurements of three individual NCs.

We already know that photopolymerization only occurs when $D>D_{\text {th }}$. By applying this condition to eq 1 , one can obtain $^{17}$

$$
\exp (-\alpha y) \geq \frac{D_{\text {th }}}{F_{\text {max }} \times D_{0}}
$$

By reducing eq 2, one can get the maximum distance $y_{\max }$ away from the $\mathrm{Au}$ NCs at which the effective dose overcomes $D_{\text {th }}$ as $^{17}$

$$
y_{\text {max }}=\alpha^{-1} \ln \left(F_{\text {max }} \times \frac{D_{0}}{D_{\text {th }}}\right)
$$

Equation 3 can be rewritten as

$$
y_{\max }=\alpha^{-1} \ln \left(F_{\max } \times f\right)
$$

thus closely fitting the logarithmic behavior of Figure 3A.

2.2. Theoretical Methods. Experimental measurements on single NCs were supported by electrodynamics simulations of the near-fields. We carried out computational electrodynamics simulations with both the finite-difference time-domain (FDTD) method ${ }^{26,27}$ and the discrete-dipole approximation (DDA).$^{28,29}$ The FDTD calculations employed the commercial software package FDTD Solutions, by Lumerical Solutions, Inc. (Vancouver, Canada), ${ }^{27}$ and the DDA calculations employed the current release of DDSCAT. ${ }^{29}$ Consistent with the experimental results, the cube sides are taken to be of length $60 \mathrm{~nm}$ and a radius of curvature of $12 \mathrm{~nm}$ is used to round all corners and sides. A multicoefficient model $\mathrm{fit}^{28}$ to the Johnson-Christy (JC) gold data ${ }^{30}$ was used in the FDTD calculations, and the JC data was used directly (or interpolated) in the case of the DDA calculations.

For the aqueous spectrum calculation, we assume the cubes are within an isotropic medium of refractive index corresponding to water, $n=1.33$. We initially considered light incident on a face of the cube with polarization oriented from side to side or oriented along the diagonal of the cube. FDTD calculations with grid spacings of $1 \mathrm{~nm}$ and DDA calculations with 200000 dipoles yielded converged results with respect to finer resolutions and were also in excellent agreement with one another both in terms of far-field spectral properties and in terms of the near-fields greater than $2 \mathrm{~nm}$ outside the cubes. Actually, DDA calculations with 100000 dipoles are sufficient if only far-field spectra are of interest. If accurate near-fields within 1-2 nm of the metal interface are desired, on the order of 400000 dipoles are necessary. Since the DDA calculations, particularly when one wavelength is of interest such as in the polymer elongation studies, can be significantly faster than the FDTD ones, most of the remaining calculations were carried out with the DDA method. With respect to the far-field extinction spectrum, we find that polarization (or equivalently cube orientation) has only a minor effect: the extinction peaks occur very close to one another, with the main difference being the magnitude of extinction maximum, which can vary somewhat (but less than 10\%). Nonetheless, for the final reported water spectrum, a Monte Carlo orientational average over 100 random cube orientations was carried out by appropriate Euler angle rotations, with a fixed incident polarization and propagation direction. See ref 31 for a new approach to orientational averaging.

The ellipsometry measurements we have carried out suggest that the polymer solution refractive index is in the $1.48-1.52$ range, very close to that of glass (1.5), and that the ITO refractive index is 1.8. Since the ITO is optically thin, the simplest approach for simulating the polymer elongation experiments is to consider a cube in a uniform medium of refractive index 1.5. Consistent with the experiments, $\lambda_{0}=532$ $\mathrm{nm}$ light in one of a variety of possible polarizations is taken to be incident from "below" the cube, and we analyze the nearfields on the top-side NC face. Figure 1 in the Supporting Information section presents schematic diagrams to help clarify the nature of these calculations. It also serves to define the polarization angle, which is the angle of the "normal" of the region in question (either a corner or side region) and the polarization angle.

\section{RESULTS AND DISCUSSION}

A quantitative study has been carried out to show how the hot spots, localized at the sharp features of the NCs, vary according to the amount of dose fraction relative to threshold dose, $f$. By measuring the polymer elongation lengths from the $\mathrm{NC}$ corner for varying dose field strengths and fitting these to an exponential function, we were able to determine the enhancement factor of the local field at the NC corner. In this case, we consider incident polarization oriented along the diagonal of the cube, i.e., along the elongation axis. We term this $\theta_{c}=0^{\circ}$ polarization referring to the angle between the outward normal from the corner region and the polarization axis (see Figure $5 B)$.

The trace of the polymer elongation at the $\mathrm{NC}$ corner as a function of $f$ is illustrated in Figure 3A, where $f$ goes from 5 to $75 \%$ of the threshold dose, in steps of 10 . This figure clearly 
shows that the NC corner exhibits an elongation of $3.5 \mathrm{~nm}$ for an incident dose $f=5 \%$, which implies that an enhancement factor of at least 20 is expected at the corner of the NC. In order to accurately determine $F_{\max }$, we have plotted in Figure $3 \mathrm{~B}$ the values of $1 / f$, deduced from $1 / f=D_{\text {th }} / D_{0}$, as a function of the polymer elongation $y$. The plot can be approximated by a single-exponential decay. ${ }^{17,32} 1 / f$ can be deduced as $1 / f=$ $F_{\max } \mathrm{e}^{-\alpha y_{\max }}$ from eq 4. Thus, when fitting the experimental data with an exponential function, one can deduce the values of the fitting parameters $F_{\max }$ and $\alpha^{-1}$ as 35 and $5 \mathrm{~nm}$, respectively.

We have also estimated $F_{\max }$ and $\alpha^{-1}$ from near-field DDA calculations. $^{29}$ In these calculations, we examine the total field intensity enhancement, $|\mathbf{E}|^{2} /\left|\mathbf{E}_{0}\right|^{2}$, in the vicinity of the top side of a cube and focus on either the region near a corner or the region near the middle of a side. Figure 4 shows maps of $|\mathbf{E}|^{2} / \mid$

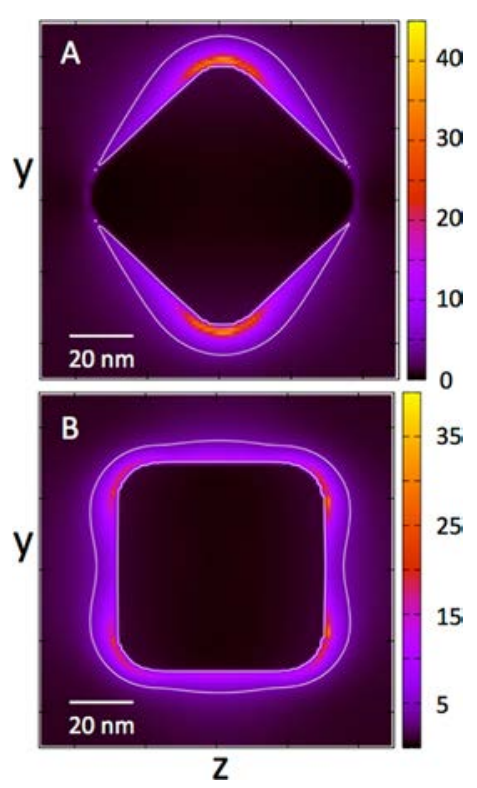

Figure 4. Top views of image maps of near-field intensity enhancements $|\mathbf{E}|^{2} /\left|\mathbf{E}_{0}\right|^{2}$ for gold NCs in an $n=1.5$ medium. In these cases, light is incident from below along the $x$-axis and is polarized along the $y$-axis (see Figure 1 in the Supporting Information). For the plots, $x$ is fixed at a value of $6 \mathrm{~nm}$ below the top of each cube. The white contour lines correspond to $|E|^{2} /\left|E_{0}\right|^{2}=4$. (A) Corner excitation with $\theta_{\mathrm{c}}=0^{\circ}$. (B) Side excitation with $\theta_{\mathrm{s}}=0^{\circ}$.

$\left.\mathbf{E}_{0}\right|^{2}$ for corner and side excitation cases with incident light propagating along the $x$-axis and polarized along the $y$-axis. In the case of light polarized along the diagonal connecting two corners $\left(\theta_{c}=0^{\circ}\right.$, Figure $\left.4 \mathrm{~A}\right)$, the largest intensity enhancements outside the cube are on the order of 45 and rapidly fall with a decay length of $2.7 \mathrm{~nm}$. Compared to the experimentally deduced $F_{\max }$ and $\alpha^{-1}$, the theoretical intensity enhancement is twice as large as $F_{\max }$ and the theoretical decay length is half as small as $\alpha^{-1}$. That is, the theoretical fields are higher and drop off more quickly than what has been experimentally deduced. However, the theoretical near-fields vary rapidly with spatial position and it is more likely that the experimentally deduced values reflect a spatial average near a given point. We averaged the theoretical results within a sphere of radius $2 \mathrm{~nm}$ about each point along the ray and obtained a maximum intensity enhancement of 30 and a length scale of $3.6 \mathrm{~nm}$, which compare more favorably with the experimental values of 35 and $5 \mathrm{~nm}$, respectively, deduced in the above paragraph. Obviously quite different results can be obtained if much larger or much smaller averaging regions were considered. The choice of a 2 $\mathrm{nm}$ radius averaging region leads to results in accord with experiment, indicating that the polymer elongation technique resolves field variations with no better than $2 \mathrm{~nm}$ resolution.

The nanoscale formation of solid polymer structures formed at the side and corner of the NC was measured using AFM at $f$ $=25 \%$. As we can see in Figures 1 and 2, the NCs present random orientations relative to the polarization of the incident laser; thus, the polymer elongations have been recorded as a function of $\theta_{\mathrm{s}}$ and $\theta_{\mathrm{c}}$. Parts $\mathrm{C}$ and $\mathrm{D}$ of Figure 5 show values of

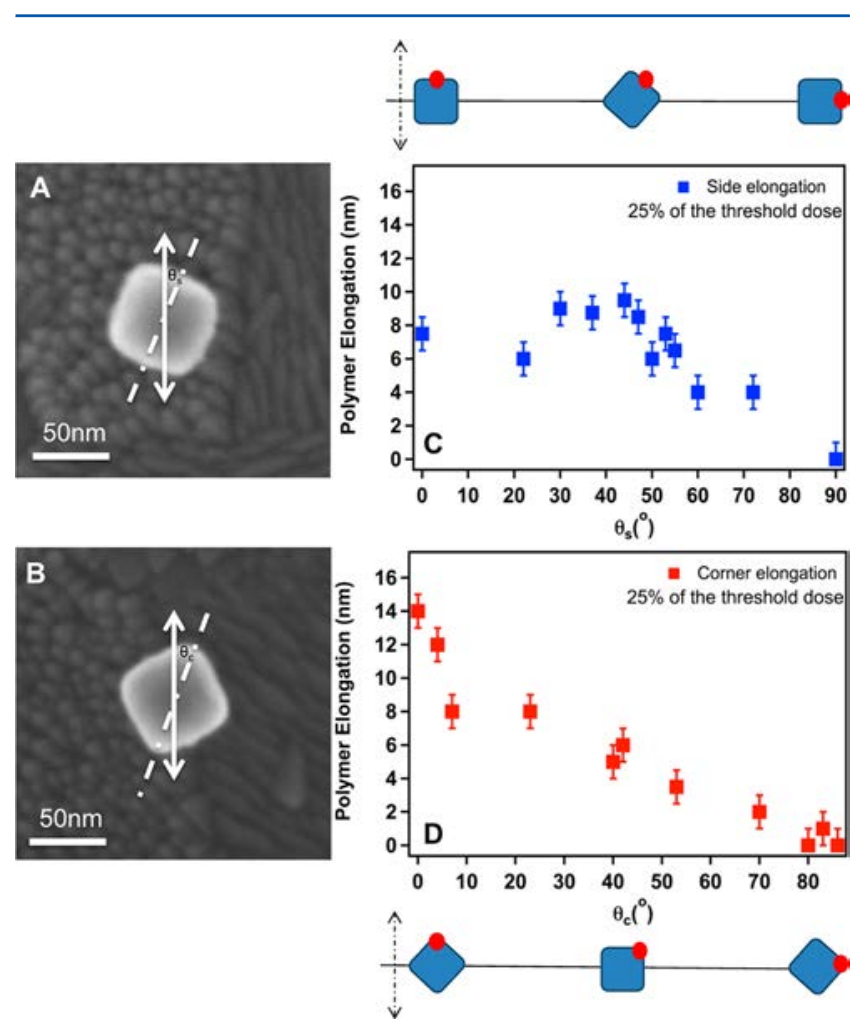

Figure 5. SEM images labeled A and B are of individual Au NCs that show $\theta_{\mathrm{s}}$ and $\theta_{\mathrm{c}}$ : (A) $\theta_{\mathrm{s}}$ is the angle between the vertical laser direction and the normal to the NC side, and (B) $\theta_{\mathrm{c}}$ is the angle between the laser polarization and the corner-corner diagonal. The white updown arrow in parts A and B denotes the polarization direction of the incident laser. Parts $\mathrm{C}$ and $\mathrm{D}$ show, respectively, the polymer elongations as a function of $\theta_{\mathrm{s}}$ and $\theta_{\mathrm{c}}$ for $D_{0}=0.25 D_{\mathrm{th}}$. Each data point is an average from measurements of three individual NCs. The error bars in parts $\mathrm{C}$ and $\mathrm{D}$ represent the minimum elongation that can be detected by the AFM, i.e., the AFM lateral resolution that is set at \pm 1 $\mathrm{nm}$.

polymer elongations of 36 similar Au NCs with 12 different orientations as compared to the laser excitation direction. The incident laser direction is kept unchanged and is shown by the up-down arrow in Figure 5A and B. This experiment was also run using seven different values of $D_{0}$ (where $f$ goes from 5 to $75 \%$ of $D_{\text {th }}$ ). For sake of brevity, we are only showing the case where $D_{0}=0.25 D_{\mathrm{th}}$. When we consider the polymer elongation for different orientations of the nanoparticles relative to the laser polarization, we observe a variation in the polymer elongation activity. This effect is due to a difference in the local field strength as the polarization of the excitation laser varies relative to the axis of the NC side and corner, as confirmed by the theoretical simulations and seen in Figure 4. 
Figure 4B corresponds to theoretical near-field intensity enhancements for side excitation; i.e., the cube and/or polarization appropriately rotated such that excitation would be between two sides and not along the diagonal. We term this case $\theta_{s}=0^{\circ}$ polarization, referring to the angle between the normal to the side (elongation direction or $y$-axis in this case) and the polarization axis (see Figure 5A). For the calculation, we simply kept the incident polarization along $y$ and rotated the cube so that the relevant near-field intensities associated with elongation from the center of a side would still be along a ray extending upward along $y$ and outside the cube. In this case, however, the intensity enhancements are less than half those associated with corner elongation. Note that, by symmetry, the $\theta_{\mathrm{c}}=\theta_{\mathrm{s}}=90^{\circ}$ cases can also be inferred from Figure 5A and $\mathrm{B}$ by a $90^{\circ}$ rotation. This corresponds to simply examining the fields in these figures along $z$, with $y$ held fixed at its central value. One then infers zero field enhancement in the corner case and some but a reduced amount in the side case. This latter feature is evidenced, for example, by inward "pinches" of the $|\mathbf{E}|^{2} /\left|\mathbf{E}_{0}\right|^{2}=4$ contour along $z$.

Parts C and D of Figure 5 exhibit that both the corner and side elongations are generally largest when the direction examined is parallel to the vertical laser polarization $\left(\theta_{\mathrm{s}}=\theta_{\mathrm{c}}=\right.$ $\left.0^{\circ}\right)$. This makes sense because the surface plasmon oscillations are then directly along those directions. A more significant polymer elongation is observed in the case of the NC corner $\left(14 \mathrm{~nm}\right.$ for $\left.\theta_{\mathrm{c}}=0^{\circ}\right)$ as compared to its side $\left(7.5 \mathrm{~nm}\right.$ for $\theta_{\mathrm{s}}=$ $\left.0^{\circ}\right)$. This is consistent with the corner excitation being stronger than the side excitation, as is evident from the theoretical nearfields (Figure 4). As the polarization angle deviates from the direction being examined, the elongations decrease and are almost zero by $\theta_{s}=\theta_{\mathrm{c}}=90^{\circ}$. The very small elongation at $\theta_{\mathrm{s}}=$ $\theta_{\mathrm{c}}=90^{\circ}$ is not surprising because $\vec{n} \perp \vec{E}_{\text {inc }}$. In the case of side excitation, there is still a surface plasmon excitation along the same side with $\theta_{\mathrm{s}}=90^{\circ}$, but it is weaker than the $\theta_{\mathrm{c}}=90^{\circ}$ case, which was also evident from the discussion of the theoretical near-fields.

Additionally, it must be noted that the polymer elongation decreases faster as $\theta_{c}$ goes from 0 to $90^{\circ}$ than $\theta_{s}$. This may reflect the fact that the corner benefits from a tip effect which is more spatially localized than the edge effect which creates enhancement at the side of the cube. It is important to note how strong the field is and how dramatically the field singularities vary at the tip of the metallic object. ${ }^{33}$ The tip effect will occur when the cube is oriented with a diagonal axis (corner to corner) parallel to the laser polarization, ${ }^{18}$ and consequently, the NC corner benefits from a higher local field strength when compared to the NC side; this also helps explain why the polymer elongation in the case of $\theta_{c}=0^{\circ}$ is greater as compared to $\theta_{\mathrm{s}}=0^{\circ}$.

We have also made estimates of the elongation lengths based on our theoretical near-field calculations. As in the discussion of Figure 4, we consider appropriate rays emanating from the cube corner or the side and take an average about each point within a sphere of radius $2 \mathrm{~nm}$. Elementary considerations suggest that, if one wishes to emulate the situation with $25 \%$ dose or $f=$ 0.25 , all electric field enhancements corresponding to $1 / f=4$ or higher will lead to photopolymerization. Note that Figure 4 also shows a white contour line corresponding to $|E|^{2} /\left|E_{0}\right|^{2}=4$. The predicted maximal elongation is therefore the maximum distance along the ray examined such that the field intensity enhancement is 4 .
Figure 6 displays the results of the calculations described above and should be compared with Figure 5C and D. There is

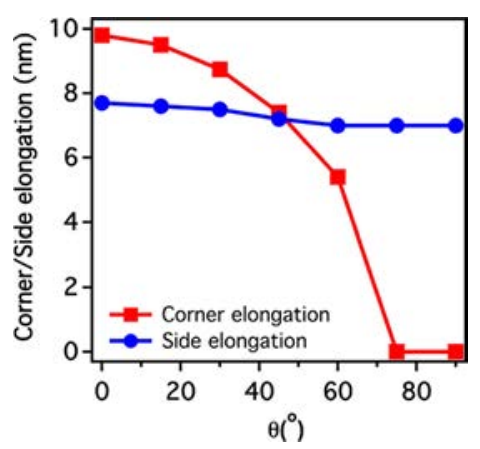

Figure 6. Theoretically calculated elongations of Au NCs as incident polarization angle changes relative to the corner (red trace) and the side (blue trace) of the cube. Note that in the case of corner elongation $\theta$ corresponds to $\theta_{\mathrm{c}}$ and in the case of side elongation $\theta$ corresponds to $\theta_{\mathrm{s}}$.

agreement between the experimental results and calculations with respect to the following points: (i) the corner elongations, at small polarization angles, are larger than the side elongations; (ii) the corner elongation, while not showing the sharp initial decrease, still decreases faster than the side elongation; (iii) the elongation lengths are on the order of $10 \mathrm{~nm}$; (iv) the side elongation is less sensitive to the polarization angle and becomes larger than the corner ones as polarization angle is increased, i.e., there is a crossover point. However, there are some noticeable discrepancies between the experimental and theoretical results, in particular: (i) the experimental corner elongation drops off more quickly at low angles and (ii) the theoretical side elongation variation with polarization angle is much weaker than the experimental one. One source of the discrepancies noted is the simple averaging technique we have used to estimate the field enhancements. We have experimented with many alternative ways of averaging the fields and have not obtained any better results. Since it is known that the surface plasmon resonance of cubes is very sensitive to fine details of the cube structure, ${ }^{4}$ this could be a factor. Simple variations in the radius of curvature, however, proved unsuccessful. We have also carried out a number of FDTD calculations to complement the DDA ones that included the slight differences of glass and polymer refractive indices and the thin ITO layer and found very similar results to those presented here on the basis of the uniform medium. It is possible that chemical effects could be playing a role such that there is not a simple linear mapping of elongation with local field intensity enhancement.

To conclude, we have shown our ability to experimentally image hot spots at the surface of Au NCs. We measured the field enhancement of a hot spot on the NC surface can be up to 35. However, there is a dramatic difference in the electromagnetic field value at the NC corner as compared to that on the side. From measurements on individual Au NCs that are oriented differently relative to the excitation laser polarization, we were able to determine the sensitivity of the near-field hot spots to incident polarization. Our experimental results were found to be consistent with computational electrodynamics calculations, although further work is required to understand the origins of some quantitative discrepancies between the theoretically estimated elongations and the experimental ones. 
The detailed characteristics of the near-field supported by the NC geometry presented here will favor the use of these nanostructures in sensing and field enhancement based applications.

\section{ASSOCIATED CONTENT}

\section{S Supporting Information}

Schemetic diagrams to help clarify the nature of the calculations. This material is available free of charge via the Internet at http://pubs.acs.org.

\section{AUTHOR INFORMATION}

\section{Corresponding Author}

*E-mail: claire.deeb@utt.fr (C.D.); renaud.bachelot@utt.fr (R.B.).

\section{Notes}

The authors declare no competing financial interest.

\section{ACKNOWLEDGMENTS}

The authors thank the Partner University Funds program (PUF 2010) for partially supporting this work. S.M. thanks the TEM team platform, DSV, CEA-Saclay, and also the Region Ile-deFrance for the financial support of their SEM. X.Z. thanks the China Scholarship Council (CSC). Use of the Center for Nanoscale Materials was supported by the U.S. Department of Energy, Office of Science, Office of Basic Energy Sciences, under Contract No. DE-AC02-06CH11357. This work was also supported by the European community FEDER fund and the Region Champagne-Ardenne: Grants HYN-NOV and NANO'MAT.

\section{REFERENCES}

(1) Coronado, E. A.; Schatz, G. C. J. Chem. Phys. 2003, 119 (7), 3926-3934.

(2) McLellan, J. M.; Li, Z.-Y.; Siekkinen, A. R.; Xia, Y. Nano Lett. 2007, 7, 1013-1017.

(3) Yi, M.; Zhang, D.; Wang, P.; Jiao, X.; Blair, S.; Wen, X.; Fu, Q.; Lu, Y.; Ming, H. Plasmonics 2011, 6 (3), 515-519.

(4) Jayabalan, J.; Singh, A.; Chari, R. Appl. Phys. Lett. 2010, 97, 041904-4.

(5) Ringe, E.; McMahon, J. M.; Sohn, K.; Cobley, C.; Xia, Y.; Huang, J.; Schatz, G. C.; Marks, L. D.; Van Duyne, R. P. J. Phys. Chem. C 2010, 114 (29), 12511-12516.

(6) Mingfang, Y.; Douguo, Z.; Xiaolei, W.; Qiang, F.; Pei, W.; Yonghua, L.; Hai, M. Plasmonics 2011, 6 (2), 213-217.

(7) Yanyun, M.; Weiyang, L.; Eun Chul, C.; Zhiyuan, L.; Taekyung, Y.; Jie, Z.; Zhaoxiong, X.; Younan, X. ACS Nano 2010, 4 (11), 67256734 .

(8) Rycenga, M.; Xia, X.; Moran, C. H.; Zhou, F.; Qin, D.; Li, Z.-Y.; Xia, Y. Angew. Chem., Int. Ed. 2011, 50, 5473-5477.

(9) Sherry, L. J.; Chang, S.-H.; Schatz, G. C.; Van Duyne, R. P.; Wiley, B. J.; Xia, Y. Nano Lett. 2005, 5 (10), 2034-2038.

(10) Galush, W. J.; Shelby, S. A.; Mulvihill, M. J.; Tao, A.; Yang, P.; Groves, J. T. Nano Lett. 2009, 9 (5), 2077-2082.

(11) Zhang, S.; Bao, K.; Halas, N. J.; Xu, H.; Nordlander, P. Nano Lett. 2011, 11, 1657-1663.

(12) Haggui, M.; Dridi, M.; Plain, J.; Marguet, S.; Perez, H.; Schatz, G. C.; Wiederrecht, G. P.; Gray, S. K.; Bachelot, R. J. ACS Nano 2012, 6, 1299-1307.

(13) Lee, Y. H.; Yan, Y.; Polavarapu, L.; Xu, Q.-H. Appl. Phys. Lett. 2009, 95, 023105 .

(14) Mahmoud, M. A.; Poncheri, A. J.; Phillips, R. L.; El-Sayed, M. A. J. Am. Chem. Soc. 2010, 132, 2633-2641.

(15) Rycenga, M.; Xia, X.; Moran, C. H.; Zhou, F.; Qin, D.; Li, Z. Y.; Xia, Y. Angew. Chem., Int. Ed. 2011, 50, 5473-5477.
(16) Ibn El Ahrach, H.; Bachelot, R.; Vial, A.; Lerondel, G.; Plain, J.; Royer, P.; Soppera, O. Phys. Rev. Lett. 2007, 98, 107402.

(17) Deeb, C.; Bachelot, R.; Plain, J.; Baudrion, A. L.; Jradi, S.; Bouhelier, A.; Soppera, O.; Jain, P. K.; Huang, L.; Ecoffet, C.; et al. ACS Nano 2010, 4, 4579-4586.

(18) Deeb, C.; Zhou, X.; Gérard, D.; Bouhelier, A.; Jain, P. K.; Plain, J.; Soppera, O.; Royer, P.; Bachelot, R. J. Phys. Chem. Lett. 2011, 2, 711.

(19) Yu, Y.; Zhang, Q.; Lu, X.; Lee, J. Y. J. Phys. Chem. C 2010, 114, 11119-11126.

(20) Sau, T. K.; Murphy, C. J. J. Am. Chem. Soc. 2004, 126, 86488649.

(21) Hubert, C.; Rumyantseva, A.; Lerondel, G.; Grand, J.; Kostcheev, S.; Billot, L.; Vial, A.; Bachelot, R.; Royer, P.; Chang, S.H.; et al. Nano Lett. 2005, 5 (4), 615-619.

(22) Soppera, O.; Jradi, S.; Lougnot, D. J. J. Polym. Sci., Part A: Polym. Chem. 2008, 46, 3783-3794.

(23) Bachelot, R.; Ecoffet, C.; Deloeil, D.; Royer, P.; Lougnot, D. Appl. Opt. 2001, 40 (32), 5860-5871.

(24) Espanet, A.; Dos Santos, G.; Ecoffet, C.; Lougnot, D. J. Appl. Surf. Sci. 1999, 1389-139, 87-92.

(25) Deeb, C.; Ecoffet, C.; Bachelot, R.; Plain, J.; Bouhelier, A.; Soppera, O. J. Am. Chem. Soc. 2011, 133, 10535-10542.

(26) Taflove, A.; Hagness, S. Computational Electrodynamics: The Finite-Difference Time-Domain Method, 3rd ed.; Artech House Publishers: 2005.

(27) Reference Guide for FDTD Solutions Release 6.5 (2009), http://www.lumerical.com/fdtd.

(28) Draine, B. Y.; Flatau, P. J. J. Opt. Soc. Am. A 1994, 11, 14911499.

(29) Draine, B. T.; Flatau, P. J. 2012, "User Guide for the Discrete Dipole Approximation Code DDSCAT (Version 7.2)”, http://arxiv. org/abs/1202.3424.

(30) Johnson, P. B.; Christy, R. W. Phys. Rev. B 1972, 6, 4367-4370.

(31) Shah, R. A.; Guyot-Sionnest, P; Gray, S. K. J. Phys. Chem. C 2012, 116, 12712-12724.

(32) Jain, P. K.; El-Sayed, M. A. J. Chem. Phys. 2010, 487, 153-164.

(33) Van Bladel, J. Singular Electromagnetic Fields and Sources; WileyIEEE Press: New York, 2002. 